\title{
Noise induces continuous and noncontinuous transi- tions in neuronal interspike intervals range
}

\author{
P R Protachevicz ${ }^{1,2}$, M S Santos ${ }^{2}$, E G Seifert ${ }^{3}$, E C Gabrick ${ }^{1}$, F S Borges ${ }^{4}$, \\ R R Borges ${ }^{5}$, J Trobia ${ }^{1,6}$, J D Szezech Jr ${ }^{1,6}$, K C Iarosz ${ }^{2,5,7, *}$, I L Caldas ${ }^{2}$, C G

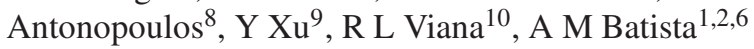 \\ ${ }^{1}$ Graduate Program in Science - Physics, State University of Ponta Grossa, 84030-900, Ponta Grossa, \\ PR, Brazil \\ ${ }^{2}$ Institute of Physics, University of São Paulo, 05508-900, São Paulo, SP, Brazil \\ ${ }^{3}$ Department of Physics, State University of Ponta Grossa, 84030-900, Ponta Grossa, PR, Brazil \\ ${ }^{4}$ Center for Mathematics, Computation, and Cognition, Federal University of ABC, 09606-045, São Bernardo \\ do Campo, SP, Brazil \\ ${ }^{5}$ Department of Mathematics, Federal University of Technology Paraná, 84016-210, Ponta Grossa, PR, \\ Brazil \\ ${ }^{6}$ Department of Mathematics and Statistics, State University of Ponta Grossa, 84030-900, Ponta Grossa, PR, \\ Brazil \\ ${ }^{7}$ Faculdade de Telêmaco Borba, FATEB, 84266-010, Telêmaco Borba, PR, Brazil \\ ${ }^{8}$ Department of Mathematical Sciences, University of Essex, CO4 3SQ, Wivenhoe Park, UK \\ ${ }^{9}$ Department of Applied Mathematics, Northwestern Polytechnical University, 710072, Xi' an, China \\ ${ }^{10}$ Department of Physics, Federal University of Paraná, 82590-300, Curitiba, PR, Brazil \\ Corresponding author: protachevicz@gmail.com.
}

\begin{abstract}
Noise appears in the brain due to various sources, such as ionic channel fluctuations and synaptic events. They affect the activities of the brain and influence neuron action potentials. Stochastic differential equations have been used to model firing patterns of neurons subject to noise. In this work, we consider perturbing noise in the adaptive exponential integrate-and-fire (AEIF) neuron. The AEIF is a two-dimensional model that describes different neuronal firing patterns by varying its parameters. Noise is added in the equation related to the membrane potential. We show that a noise current can induce continuous and noncontinuous transitions in neuronal interspike intervals. Moreover, we show that the noncontinuous transition occurs mainly for parameters close to the border between tonic spiking and burst activities of the neuron without noise.
\end{abstract}

noise, adaptive exponential integrate-and-fire, neuronal activities

\section{Introduction}

Neuronal activities play an important role in brain functions. In the late 1800s, Caton [1] and Beck [2] recorded electrical brain activities in various animal species by means of electrodes. Hans [3] discovered the electroencephalography (EEG) in the 1920s and made the first electrocorticogram in a human. Since then, EEG has been used to measure electrical activities generated by neuronal action potentials [4, 5]. 
Mathematical models have been considered to reproduce neuronal action potentials. In 1907, Lapicque [6] developed a neuron model based on an electric circuit composed of a capacitor and resistor in parallel [7]. Hodgkin and Huxley [8] proposed in 1952 a model containing terms related to different types of ion channels. In 2005, Brette and Gerstner [9] introduced the adaptive exponential integrate-and-fire (AEIF) model. AEIF is a model that has an exponential spiking mechanism combined with an adaptation equation [10]. Neuronal networks composed of AEIF neurons have been used to study synchronous behaviours [11], self-sustained activity [12], and firing patterns [13]. Shiau and Buhry [14] analysed interneuronal gamma oscillations in the hippocampus by means of AEIF neurons.

The activity of isolated neurons can be affected by different types of noise. There are many sources of noise in the brain, such as from genetic processes, thermal noise, ionic channel fluctuations, and synaptic events [15]. It was reported that noisy neurons are critical for learning [16]. Synaptic noise is a source of randomness in the neuronal interspike intervals [17]. Brunel et al. [18] studied the effects of synaptic noise on the frequency response of neurons. They found that noise inputs can enhance high frequency responses. González-Villar et al. [19] observed that higher neuronal noise can be related to cognitive dyscognition in chronic pain syndromes. It was reported that Lévy noise [20, 21, 22] can induce stochastic resonance in the FitzHugh-Nagumo (FHN) neuron [23], as well as temporal and spatial coherence in coupled FHN neurons [24].

Neuronal activities with noise have been modelled by means of stochastic differential equations [25, 26]. Various stochastic neuron models were introduced, for instance the stochastic integrate-and-fire [27] and Fitzhugh-Nagumo [28] neuronal models. The stochastic Hodgkin-Huxley model [29], where the noise is incorporated in the ion channel, is able to simulate the behaviour of cerebellar granule cells [30]. Braun et al. [31] studied a Hodgkin-Huxley type model subjected to a current noise. They found transitions from single to grouping of spikes in response to temperature alterations.

In this work, we include noise in the AEIF model and study the effects on neuronal firings. To do that, we compute the neuronal interspike intervals and calculate their coefficient of variation. The coefficient of variation can be used to measure the variability of the interspike intervals and to identify tonic spiking and burst patterns. By varying the noise amplitude, we show the existence of continuous and noncontinuous transitions in the neuronal interspike intervals. The noncontinuous transitions occur mainly for parameters close to the border between tonic spiking and burst activities of the neuron without noise.

The paper is organized as follows. In Section 2, we introduce the stochastic model that mimics neuronal activity and the diagnostic tools. Section 3 presents our results about the effects of noise on neuronal activities. Finally, we draw our conclusions in the last section. 


\section{Methodology}

\subsection{Stochastic neuron model}

The adaptive exponential integrate-and-fire (AEIF) model has been used to mimic neuronal tonic spiking and burst activities. In this work, We consider a stochastic adaptive exponential integrate-and-fire (SAEIF) model, where a stochastic term, which corresponds to a noise current input, is added to the AEIF model. The SAEIF neuron model is given by

$$
\begin{aligned}
\frac{d V}{d t} & =\frac{1}{C_{\mathrm{m}}}[f(V)-w+I]+\zeta(t), \\
\frac{d w}{d t} & =\frac{1}{\tau_{m}}\left[a\left(V-E_{\mathrm{L}}\right)-w\right]
\end{aligned}
$$

where $V$ and $w$ correspond to the membrane potential and the adaptation current of a single neuron, respectively. $f(V)$ is a function defined as

$$
f(V)=-g_{\mathrm{L}}\left(V-E_{\mathrm{L}}\right)+g_{\mathrm{L}} \Delta_{\mathrm{T}} \exp \left(\frac{V-V_{\mathrm{T}}}{\Delta_{\mathrm{T}}}\right) .
$$

In our simulations, we consider $C_{\mathrm{m}}=200 \mathrm{pF}$ (membrane capacitance), $g_{\mathrm{L}}=12 \mathrm{nS}$ (leak conductance), $E_{\mathrm{L}}=-70 \mathrm{mV}$ (resting potential), $\Delta_{\mathrm{T}}=2 \mathrm{mV}$ (slope factor), $V_{\mathrm{T}}=-50 \mathrm{mV}$ (threshold potential), $\tau_{w}=300 \mathrm{~ms}$ (adaptation time constant), and $a=2$ $\mathrm{nS}$ (level of subthreshold adaptation). A constant current $I$ equal to $500 \mathrm{pA}$ is injected to the neuron. We add a noise source $\zeta(t)$, where $\langle\zeta(t)\rangle=0$ and $\left\langle\zeta(t) \zeta\left(t^{\prime}\right)\right\rangle=$ $2 D \delta\left(t-t^{\prime}\right) . \quad D$ is the noise amplitude and $\delta()$ is the Dirac delta function. We consider a refractory period of $1 \mathrm{~ms}$, namely a time in which the neuron can not fire. In the refractory period, the membrane voltage is maintained in the reset potential value $V_{\mathrm{r}}$. When the neuronal membrane potential is above a threshold $\left(V>V_{\text {thres }}\right)$ [10], the variable states are updated according to

$$
\begin{aligned}
& V \rightarrow V_{\mathrm{r}}, \\
& w \rightarrow w_{\mathrm{r}}=w+b,
\end{aligned}
$$

where $V_{\mathrm{r}}$ and $b$ are the reset potential and the triggered adaptation addition, respectively. The set of equations are solved by means of the Stochastic Runge-Kutta algorithm [32].

\subsection{Diagnostic tools}

Aiming to quantify the variability of the neuronal firings, we compute the interspike intervals (ISI). The $m$-th ISI is defined as

$$
\mathrm{ISI}_{m}=t_{m+1}-t_{m}
$$

where $t_{m}$ is the time of the $m$-th neuronal fire. The coefficient of variation of ISI is then defined by

$$
\mathrm{CV}=\frac{\sigma_{\mathrm{ISI}}}{\overline{\mathrm{ISI}}}
$$


where $\sigma_{\text {ISI }}$ is the standard deviation and $\overline{\text { ISI }}$ the mean value of ISI. In the absence of noise $(D=0)$, the neuron in Eq. 1 can exhibit tonic spiking and burst activities characterised by $\mathrm{CV}<0.5$ and $\mathrm{CV} \geq 0.5$, respectively [11, 33, 34]. The noise increases the irregularity of the firing times, and as a consequence, for intense noise amplitude, $\mathrm{CV},<\approx 1$ for neuronal tonic spiking and $\mathrm{CV}>1$ for burst patterns [35].

In this work, to identify bifurcations in the range of the ISIs values, we compute the minimal ISI ${ }^{\min }$ and maximal ISI ${ }^{\max }$ value of the ISI in the parameter space $b \times V_{\mathrm{r}}$. For $D=0$, we identify a region where $\left(\mathrm{ISI}^{\mathrm{max}}-\mathrm{ISI}^{\mathrm{min}}\right) \leq 0.5 \mathrm{~ms}$. When $D>0$, this difference increases and belongs to the same regions if the new ISI (ISI new $)$ satisfies the inequality

$$
\left[\operatorname{ISI}_{j}^{\min }-\operatorname{tol}(D)\right] \leq \operatorname{ISI}_{\text {new }} \leq\left[\operatorname{ISI}_{j}^{\max }+\operatorname{tol}(D)\right],
$$

where the tolerance function (tol) is given by

$$
\operatorname{tol}(D)=95 \cdot D^{0.25} \text {. }
$$

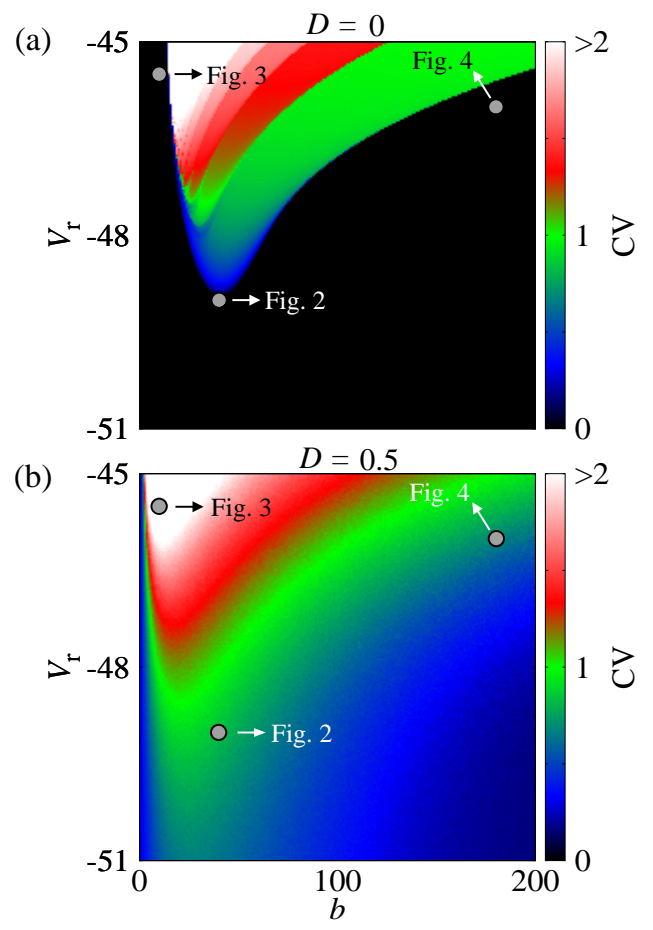

Figure 1: Parameter space $V_{\mathrm{r}} \times b$ for (a) $D=0$ and (b) $D=0.5$, where the colour bar corresponds to the $\mathrm{CV}$ values.

When ISI $I_{\text {new }}$ does not satisfy Eq. (7), a noncontinuous transition occurs in the ISI, and as a consequence, $j$ new regions with different ISI ranges,

$$
\left(\mathrm{ISI}_{\text {new }}^{\max }-\mathrm{ISI}_{\text {new }}^{\min }\right) \leq 40 \mathrm{~ms},
$$


appear. Regions larger than $40 \mathrm{~ms}$ are not considered as discrete transitions, as well as regions with a small number of visits $(<10)$ are not considered when identifying new regions.

\section{Results}

The coefficient of variation $(\mathrm{CV})$ has been used to identify tonic spiking and burst patterns. Figure 1 shows the $\mathrm{CV}$ values (colour bar) in the parameter space $V_{\mathrm{r}} \times b$ for (a) $D=0$ and (b) $D=0.5$. Increasing $D$ from 0 (Fig. 1 (a)) to 0.5 (Fig. 1 (b)), increases the values of $\mathrm{CV}$ above 0 . The changes in $\mathrm{CV}$ when $D$ increases, indicate alterations in the dynamical behaviour. Due to this fact, we compute ISI $_{m}$ for different values of $V_{\mathrm{r}}$ and $b$ to understand the noise effect on neuronal dynamics.
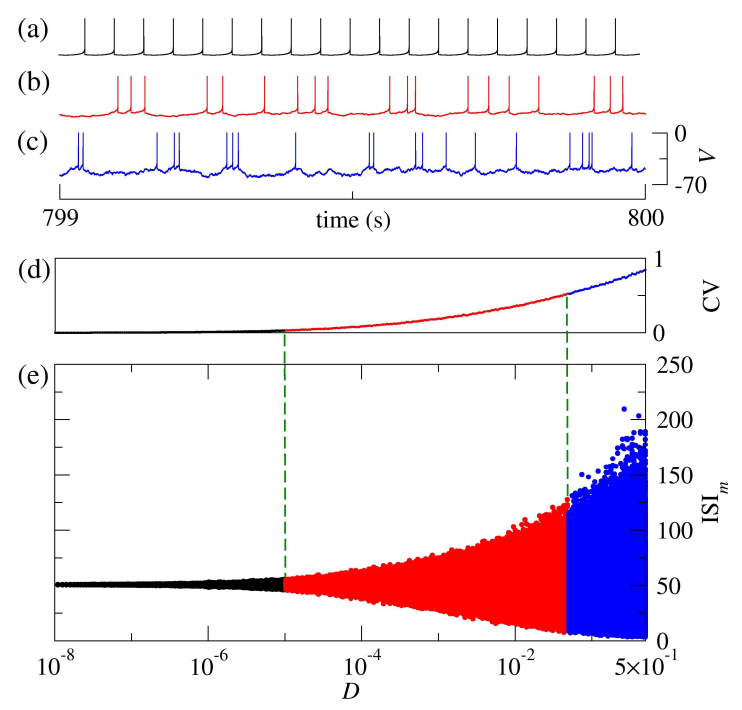

Figure 2: Temporal evolution of $V$ for (a) $D=0$, (b) $D=0.05$, and (c) $D=0.5$ for $V_{\mathrm{r}}=-49 \mathrm{mV}$ and $b=40 \mathrm{pA}$. (d) CV and (e) $\operatorname{ISI}_{m}$ as a function of $D$.

For $V_{\mathrm{r}}=-49 \mathrm{mV}, b=40 \mathrm{pA}$, and without noise $(D=0)$, the neuron exhibits tonic spiking activities with an ISI value approximately equal to $50 \mathrm{~ms}$ (see Fig. 22(a)). Depending on the noise amplitude, the neuronal pattern can display tonic spiking or burst activities, as shown in panels (b) and (c) in Fig. 2, respectively, and as a consequence, CV increases (Fig. 2(d)). In Fig. 2(e), we observe a continuous increase of the ISI range when the noise amplitude is increased from $10^{-8}$ to 0.5 . Figure 3 displays a situation in which a noncontinuous change in the values of the ISI range occurs, where we consider $V_{\mathrm{r}}=-45.5 \mathrm{mV}$ and $b=10 \mathrm{pA}$. The neuron exhibits tonic spiking activity for $D=0$ with ISI value approximately equal to $8 \mathrm{~ms}$ (Fig. 3(a)). The tonic spiking activities turn into bursts for $D=0.05$ (Fig. 3 (b)) and $D=0.5$ (Fig. 3(c)), and as a result, the $\mathrm{CV}$ increases, as shown in Fig. 3(d). We find that for $D \approx 10^{-4}$, a new ISI 

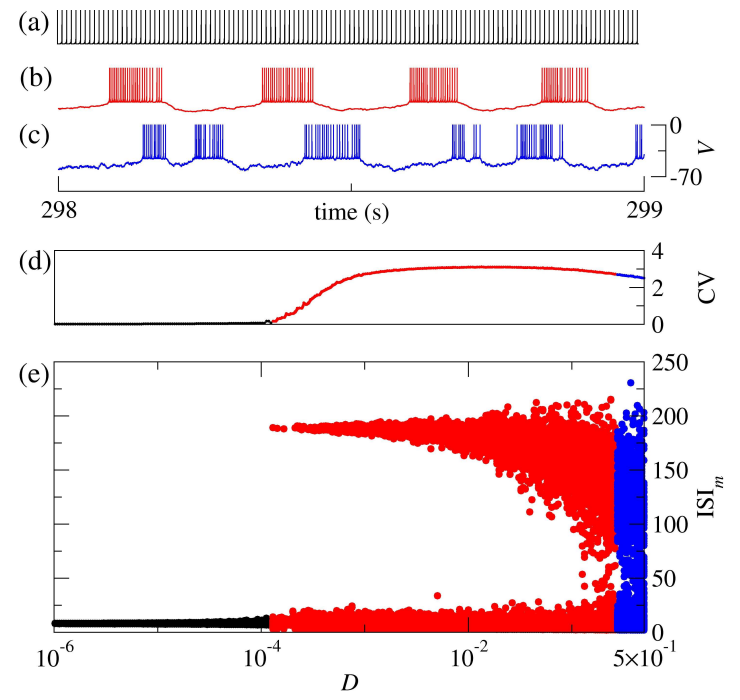

Figure 3: Temporal evolution of $V$ for (a) $D=0$, (b) $D=0.05$, and (c) $D=0.5$ for $V_{\mathrm{r}}=-45.5 \mathrm{mV}$ and $b=10 \mathrm{pA}$. (d) CV and (e) $\operatorname{ISI}_{m}$ as a function of $D$.

range appears $(\approx 190 \mathrm{~ms})$, namely the noise current induces a noncontinuous transition from 1 to 2 ISI ranges $(1 \rightarrow 2)$.

The unperturbed AEIF neuron exhibits tonic spiking activities for $V_{\mathrm{r}}=-46 \mathrm{mV}$ and $b=180 \mathrm{pA}$ (Fig. 4(a)). Without noise, the interval time between tonic spiking activities is periodic, however a small noise amplitude is enough to alter the time interval of irregular behaviour, as shown in panels (b) and (c) in Fig. 4. Moreover, the noise also increases the values of CV (Fig. 4(d). The increase of CV begins when the $1 \rightarrow 3$ transition occurs (Fig. 4(e)).

In Fig. 5(a), we identify the regions in the parameter space where the continuous (white) and noncontinuous transitions (other colours) appear. In our simulations, we find the following noncontinuous transitions of the ISI range: $1 \rightarrow 2$ (orange), $1 \rightarrow 3$ (gray), $2 \rightarrow 3$ (red), $2 \rightarrow 4$ (blue), $3 \rightarrow 4$ (green), and $3 \rightarrow 5$ (black). Figure 5b) displays the magnification of the parameter space in the intervals $V_{\mathrm{r}}=[-46,-45.5]$ $\mathrm{mV}$ and $b=[50,100] \mathrm{pA}$. The transition is identified through 50 repetitions with an analysed time equal to $25 \mathrm{~s}$, where the transient time is set equal to $1 \mathrm{~s}$.

\section{Conclusions}

Neurons are nerve cells responsible for receiving and transmitting information in the brain. Mathematical models have been developed and used to describe neuronal behaviour. The adaptive exponential integrate-and-fire (AEIF) model has been considered to mimic neuronal tonic spiking and burst activities. In this work, we include a stochastic term in the AEIF model to analyse the effect of noise in the activity of the neuron. The noise is inherent to neuronal activities and arise from several sources. 

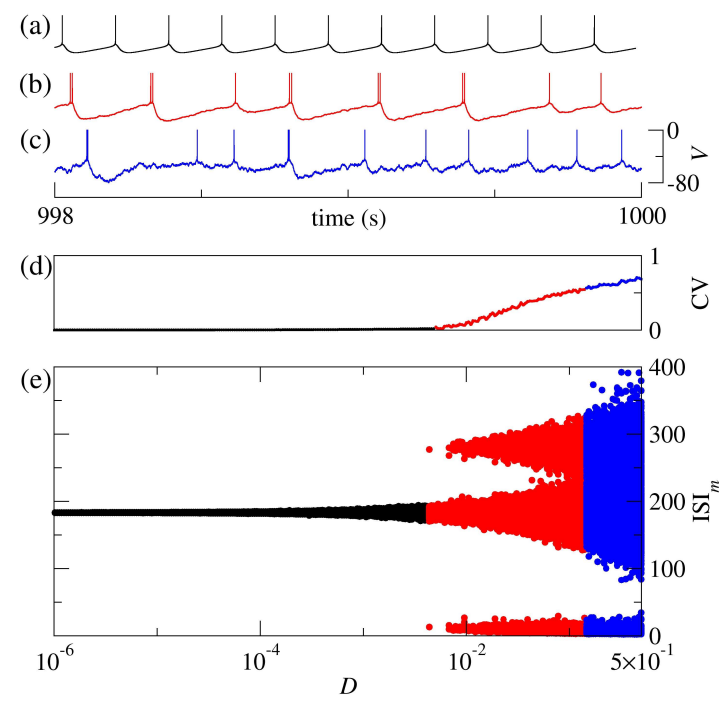

Figure 4: Temporal evolution of $V$ for (a) $D=0$, (b) $D=0.05$, and (c) $D=0.5$ for $V_{\mathrm{r}}=-46 \mathrm{mV}$ and $b=180 \mathrm{pA}$. (d) CV and (e) $\operatorname{ISI}_{m}$ as a function of $D$.

We find that noise induces alteration in the interspike interval (ISI), and as a consequence, the neuronal activities can change from tonic spiking to burst activites and vice versa. We observe that bifurcations in the ISI range occur when the noise amplitude is increased. The bifurcations are noncontinuous transitions and depend not only on the noise amplitude, but also on the reset potential $\left(V_{\mathrm{r}}\right)$ and the triggered adaptation addition $(b)$. We find the noncontinuous bifurcations in the regions of the parameter space $V_{\mathrm{r}} \times b$ close to the border in which the unperturbed neuron changes from tonic spiking to burst patterns. In the parameter space with burst pattern, we also observe discrete bifurcations close to the border in which unperturbed neurons exhibit different number of fires per burst. Therefore, noise plays an important role in the neuronal behaviour related to tonic spiking and burst activities, as well as to ISI ranges.

\section{Acknowledgment}

This study was financially supported by the following Brazilian government agencies: Fundação Araucária, National Council for Scientific and Technological Development, Coordination for the Improvement of Higher Education Personnel, and São Paulo Research Foundation (2015/07311-7, 2017/18977-1, 2018/03211-6).

\section{References}

[1] R Caton, Brit. Med. J. 2278 (1875)

[2] A Coenen and O Zayachkivska, Adv. Cogn. Psychol. 9216 (2013) 


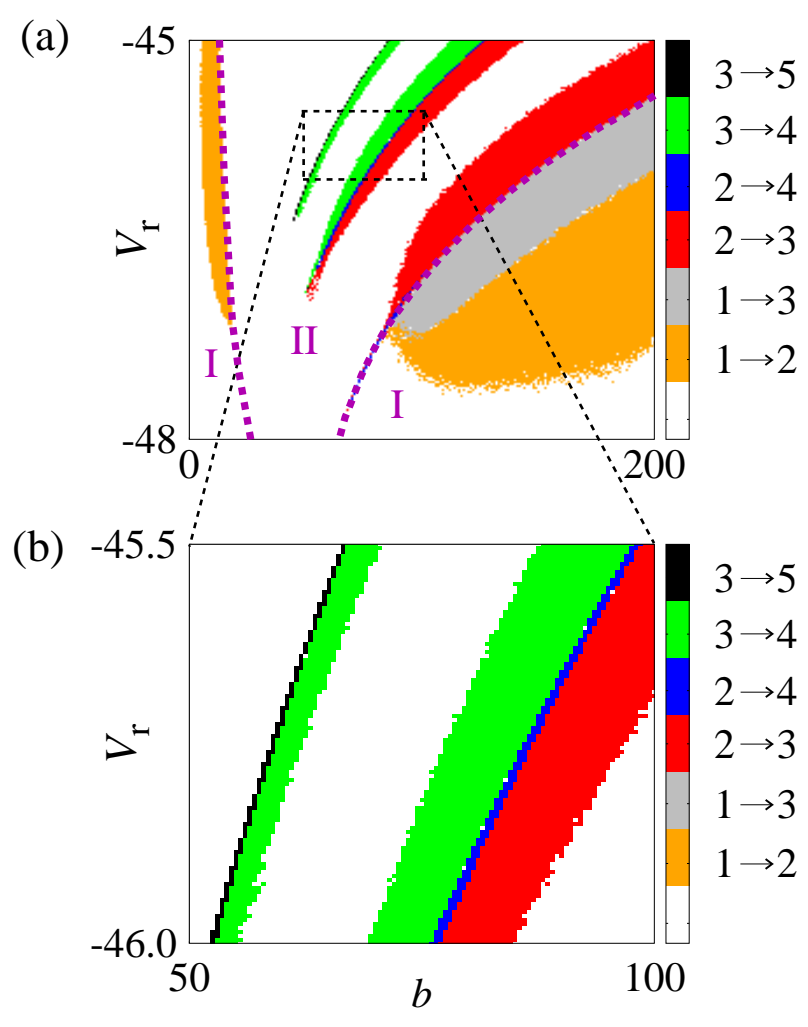

Figure 5: (a) $V_{\mathrm{r}} \times b$ showing the regions where continuous (white) and noncontinuous transitions of the ISI range appear: $1 \rightarrow 2$ (orange), $1 \rightarrow 3$ (gray), $2 \rightarrow 3$ (red), $2 \rightarrow 4$ (blue), $3 \rightarrow 4$ (green), and $3 \rightarrow 5$ (black). (b) Magnification of the parameter space in the intervals $V_{\mathrm{r}}=[-46,-45.5] \mathrm{mV}$ and $b=[50,100] \mathrm{pA}$. Regions I and II correspond to tonic spiking and burst activities, respectively.

[3] T J La Vaque, J. Neurother. 31 (1999)

[4] L I Zhang and M-M Poo, Nat. Neurosci. 41207 (2001)

[5] J C Henry, Neurology 672092 (2006)

[6] L Lapicque, J. Physiol. Pathol. Gen. 9620 (1907)

[7] A N Burkitt, Biol. Cybern. 951 (2006)

[8] A L Hodgkin and A F Huxley, J. Physiol. 116449 (1952)

[9] R Brette and W Gerstner, J. Neurophysiol. 943637 (2005)

[10] R Naud, N Marcille, C Clopath and W Gerstner, Biol. Cybern. 99335 (2008) 
[11] F S Borges, P R Protachevicz, E L Lameu, R C Bonetti, K C Iarosz, I L Caldas, M S Baptista and A M Batista, Neural Netw. 901 (2017)

[12] F S Borges, P R Protachevicz, R F O Pena, E L Lameu, G S V Higa, A H Kihara, F S Matias, C G Antonopoulos, R de Pasquale, A C Roque, K C Iarosz, P Ji and A M Batista, Physica A 537122671 (2020)

[13] M S Santos, P R Protachevicz, K C Iarosz, I L Caldas, R L Viana, F S Borges, H P Ren, J D Szezech Jr, A M Batista and C Grebogi, Chaos 29043106 (2019)

[14] L Shiau and L Buhry, Neurocomputing 331220 (2019)

[15] A A Faisal, L P J Selen and D M Wolpert, Nat. Rev. Neurosci. 9292 (2008)

[16] T A Engel, W Chaisangmongkon, D J Freedman and X-J Wang, Nat. Commun. 66454 (2015)

[17] W H Calvin and C F Stevens, J. Neurophysiol. 31574 (1968)

[18] N Brunel, F S Chance, N Fourcaud and L F Abbott, Phys. Rev. Lett. 862186 (2001)

[19] A J González-Villar, N Samartin-Veija, M Arias and M T Carrillo-de-la-Peña, Sci. Rep. 75841 (2017)

[20] Y Xu, J Feng, J J Li and H Q Zhang, Chaos 23013110 (2013)

[21] Y Xu, J Feng, W Xu and R C Gu, CMES 106309 (2015)

[22] Y Xu, Y G Li, H Zhang, X F Li and J Kurths, Sci. Rep. 631505 (2016)

[23] W ZhanQing, X Y Yong and Y Hui, Sci. China Technol. Sc. 59371 (2016)

[24] Z Wang, Y Xu, Y Li and J Kurths, J. Stat. Mech. Theory Exp. 2019103501 (2019)

[25] J Wu, Y Xu and J Ma, PLoS ONE 12 e0174330 (2017)

[26] J Feng, W Xu, Y Xu and X L Wang, Physica A 531121747 (2018)

[27] P Lansky, P Sanda, J. Comput. Neurosci. 21211 (2006)

[28] H C Tuckwell, R Rodriguez and F Y M Wan, Neural Comput. 15143 (2003)

[29] J H Goldwyn, N S Imennov, M Famulare and E Shea-Brown, Phys. Rev. E 83 041908 (2011)

[30] A Saarinen, M-L Linne and O. Yli-Harja, Neurocomputing 691091 (2006)

[31] H A Braun, J Schwabedal, M Dewald, C Finke, S Postnova, M T Huber, B Wollweber, H Schneider, M C Hirsch, K Voigt, U Feudel and F Moss, Chaos 21047509 (2011)

[32] R L Honeycutt, Phys. Rev. A 45 (1992) 600 (1992) 
[33] P R Protachevicz, R R Borges, A S Reis, F S Borges, K C Iarosz, E L Lameu, E E N Macau, R L Viana, I M Sokolov, F A S Ferrari, J Kurths, A M Batista, C-Y Lo, Y He and C-P Lin, Physiol. Meas. 39074006 (2018)

[34] P R Protachevicz, F S Borges, E L Lameu, P Ji, K C Iarosz, A H Kihara, I L Caldas, J D Szezech Jr, M S Baptista, E E N Macau, C Antonopoulos, A M Batista and J Kurths, Front. Comput. Neurosc. 131 (2019)

[35] W Gerstner, W N Kistler, R Naud and L Paninski, Neuronal Dynamics: From single neurons to networks and models of cognition, Cambridge University Press (2014) 